\title{
Method for controlling boundary condition effects on the measurement of acoustic properties of small samples in tubes
}

Thomas Dupont ${ }^{\mathrm{a}^{*}}$, Kévin Verdière ${ }^{\mathrm{b}}$, Philippe Leclaire ${ }^{\mathrm{c}}$, Raymond Panneton ${ }^{\mathrm{d}}$,

${ }^{a}$ Department of Mechanical Engineering, École de Technologie Supérieure, 1100 rue Notre-Dame Ouest, Montréal, Que., Canada H3C 1K3

${ }^{\mathrm{b}}$ MECANUM Inc, 2444 Rue Bonin, Sherbrooke, Que., Canada J1K 1C4

${ }^{c}$ DRIVE EA1859, Univ. Bourgogne Franche Comté, ISAT, BP 31 - 49 rue Mlle Bourgeois 58027

Nevers - France

${ }^{\mathrm{d}}$ CRASH-UdeS, Centre de recherche acoustique-signal-humain de l'Université de Sherbrooke, 2500 boul. de l'Université, Sherbrooke, Que., Canada J1K 2RI

${ }^{*}$ Corresponding author:

Tel.: +1 514 396-8771; Fax: +1-514 396-8530

"E-mail address: thomas.dupont@etsmtl.ca 
T. Dupont et al.

\begin{abstract}
As standard ASTM E2611 reveals, the normal incidence sound transmission loss measured on a small sample in an acoustic tube is not only a property of the material, but it is also strongly dependent on boundary conditions (generally unknown) and on the way the material is mounted. This article proposes an experimental method to control the effects of the lateral boundary conditions in an acoustic tube. The main objective is to deduce the properties of a "client element" (material sample) from the measured global acoustic properties of a patchwork composed by the "client material" and a known "host support". Three patchwork configurations have to be distinguished: patchworks with and without an impervious and rigid interface between the elements, and patchworks composed by elements which cannot be identified as equivalent fluids. For each of these configurations, it is proposed to use a specific method based on the Mixing Rule Method (MRM) or on the Parallel Transfer Matrix Methods (P-TMM or dP-TMM) used in reverse way. Numerical and experimental validations are proposed in acoustic tubes on convenient configuration: a material sample surrounded by an air ring. This configuration allows reducing the material elasticframe behavior to leave a limp-frame behavior. The proposed methods allow removing the effect of the lateral air ring host surrounding the material. For homogeneous materials the two methods based on MRM and dP-TMM give similar good results. For non-homogeneous materials or for materials which cannot be modelled as equivalent fluids only the method based on AP-TMM gives good results.
\end{abstract}

Keywords: acoustic tube, impedance tube, lateral boundary conditions, sound absorption, sound transmission, acoustic characterization, Mixing Rule Method, Parallel-Transfer Matrix Method, parallel element patchwork 


\section{T. Dupont et al.}

\section{Introduction}

ASTM E $2611^{1}$ is a standard test method for the measurement of the normal incidence sound transmission of acoustical materials based on the Transfer Matrix Method (TMM). In this method, a small finite lateral size sample is placed in a tube which contains one loudspeaker and microphones. As indicated in ASTM E2611 ${ }^{1}$, "transmission loss is not only a property of a material, but it is also strongly dependent on boundary conditions inherent in the method and details of the way the material is mounted". ASTM E2611 ${ }^{1}$ also states that "each sample must have the same shape and area as the tube cross-section", and that a "small opening around the edge will have a dramatic impact on the transmission loss calculations. Any peripheral cracks or gaps must be sealed with petroleum jelly, modelling clay, or putty." Note that standards ISO $10534-2^{2}$ and ASTM E1050-90 ${ }^{3}$ on the measurement of normal incidence sound absorption coefficient in an acoustic tube propose similar remarks and recommendations. For some materials, it is very difficult to cut the sample exactly in the same shape and area as the tube cross-section. This yields a circumferential air gap or edge constraint between the sample and tube wall. Several studies ${ }^{4-15}$ have shown that the mounting conditions (circumferential edge constraint ${ }^{1,4-10}$ and circumferential air gap $^{11-15}$ ) can significantly alter acoustic tube measurements.

The motivation for this work comes from our desire to obtain the normal incidence acoustic properties of a small sample of material weakly, if at all, affected by its circumferential boundary conditions during an acoustic tube measurement. It would be as if the measurement had been made under an acoustic plane wave excitation at normal incidence on a sample of infinite lateral dimensions. Consequently, the objective of this paper is to propose an experimental method to 


\section{T. Dupont et al.}

improve the measurement accuracy of the transfer matrix and transmission loss of a material sample independent from its lateral boundary conditions.

To meet this objective, an analytical method based on a particular mounting configuration of a small material sample in an acoustic tube is proposed to retrieve the normal incidence acoustic properties of a material free, or almost free, from circumferential boundary condition effects. The method consists in measuring the transfer matrix and acoustic properties of a client element when it is tested in parallel with a host element in an acoustic tube following ASTM E2611. Here, the client element is the material to test (with unknown acoustic properties), while the host element is acoustically defined (e.g., air). The analytical method uses three different approaches which are based on homogenization, namely the parallel transfer matrix method ${ }^{16-19}(\mathrm{P}-\mathrm{TMM})$ and it $\operatorname{extension}^{19}$ (P-TMM with discretization or dP-TMM), and the Mixing Rule Method ${ }^{20-23}$ (MRM). These methods allows studying acoustic materials in the form of patchworks (i.e., assemblies of materials in parallel). In this paper, it is proposed to use these methods in reverse configuration that is to say from the measured global transfer matrix of a patchwork to the transfer matrix of the client element.

In section 2 of this paper, the three approaches are described. In section 3, the proposed method is validated for a two-element patchwork (material samples with peripheral air layer) based on finite element simulations of virtual acoustic tube measurements, the limitation of the proposed method is discussed. In section 4, the method is applied on real acoustic tube measurements performed on different two-element patchworks (material samples with peripheral air layer). Section 5 discusses the results of the proposed method. Section 6 presents practical advice and gives recommendations 


\section{T. Dupont et al.}

on the use of the method. Finally, a conclusion is given. Parts of this article were presented at conferences. ${ }^{24,25}$

\section{Materials and methods}

\subsection{Description of the patchwork}

In this study, a two-element patchwork (subscript $p$ ) composed of a host element (subscript $h$ ) and a client element (subscript $c$ ) is considered. The surface area ratio (SAR) of the client element is $r_{c}$ and that of the host element is the complement $r_{h}=1-r_{c}$. The SAR is the ratio of the crosssection area of the client element to the total area of the patchwork. The patchwork has the same shape and size as the tube. Its two elements have the same thickness, and their shape and size remain the same over the entire thickness. An illustration of the patchwork mounted in a three-microphone instrumented transmission tube is given in Figure 1. The details of the transmission tube and the underlying measurement method are presented in Section 4. 
T. Dupont et al.

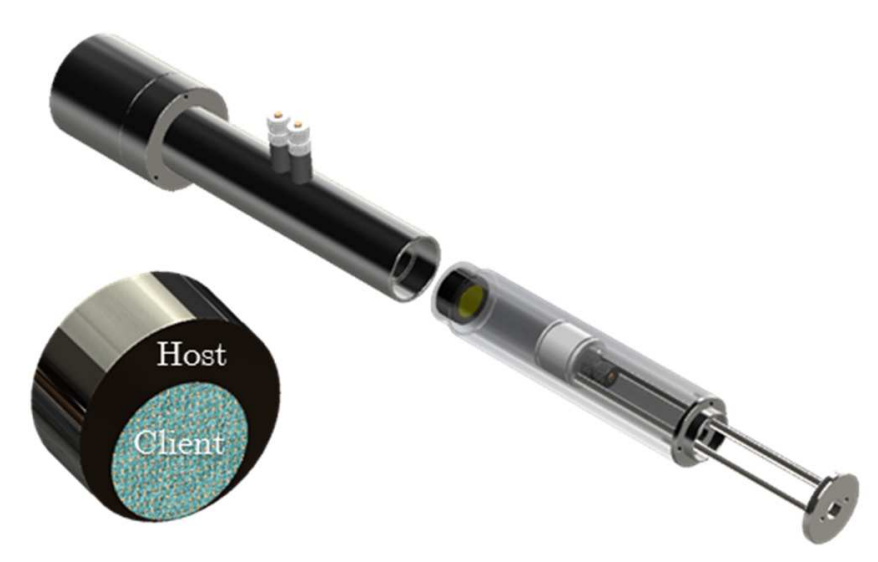

Figure 1 : Illustration of the two-element patchwork mounted in a three-microphone instrumented transmission tube. The patchwork is composed of a client and a host element. The rigid piston can slide into the tube to create air cavities of different depths behind the patchwork.

The client element is a sample of the material whose acoustic properties are to be measured at normal incidence. These properties are not intended to be affected by circumferential boundary conditions; as if they had been measured on a sample of infinite lateral dimensions. The client element can have any cross-sectional shape, but it retains its shape along the thickness. Only the SAR is important. Typically, the client element is a single-layer homogeneous porous material that can be acoustically modelled as an equivalent fluid. ${ }^{26}$ However, it can also be a material that cannot be modelled as an equivalent fluid (e.g., a non-homogeneous multilayer material). These two situations will be tested in section IV.

The host element is typically a ring surrounding the client element. It is typically a solid or air; however it could also be of another type. For instance, it could be a porous material that can be modelled or not as an equivalent fluid. In this paper, only the case of air will be studied. The case of a solid or porous hosts has been treated elsewhere. ${ }^{24,25}$ 


\section{T. Dupont et al.}

The patchwork configuration may be of three types. The first type corresponds to a patchwork where there is no fluid exchange at the interface between the host element and client element. In other word, the client-host interface is impervious. A particular case of this type is configuration with a solid ring surrounding the client as shown in Figure 1, this particular configuration represents a surface reduction in the tube that has already been studied elsewhere. ${ }^{24,25}$ The second configuration corresponds to a patchwork where the interface between the host element and the client element allows fluid exchange. In other word, the client-host interface is pervious. In this second configuration, both the client element and host element can be acoustically modelled as an equivalent fluid. The third configuration is a variation of the second configuration, where at least one of patchwork element (typically the client) cannot be modelled as an equivalent fluid. The second and third configurations will be tested in sections III and IV when the host element is an air ring surrounding the client element. These are the most convenient and simple configurations for an experimental dissemination of the method.

\subsection{General description of the method}

For each of the three aforementioned patchwork configurations, a specific approach is proposed. For the first configuration, the proposed approach uses the Parallel Transfer Matrix Method ${ }^{16-19}$ $(\mathrm{P}-\mathrm{TMM})$ in a reverse way. For the second configuration, the Mixing Rule Method ${ }^{20-23}(\mathrm{MRM})$ is used in a reverse way. For the third configuration, the extension of P-TMM ${ }^{19,27}$ with discretization $(\mathrm{dP}-\mathrm{TMM})$ is used in a reverse way. Figure 2 summarizes the global method and its three approaches depending on the type of configuration. 
T. Dupont et al.

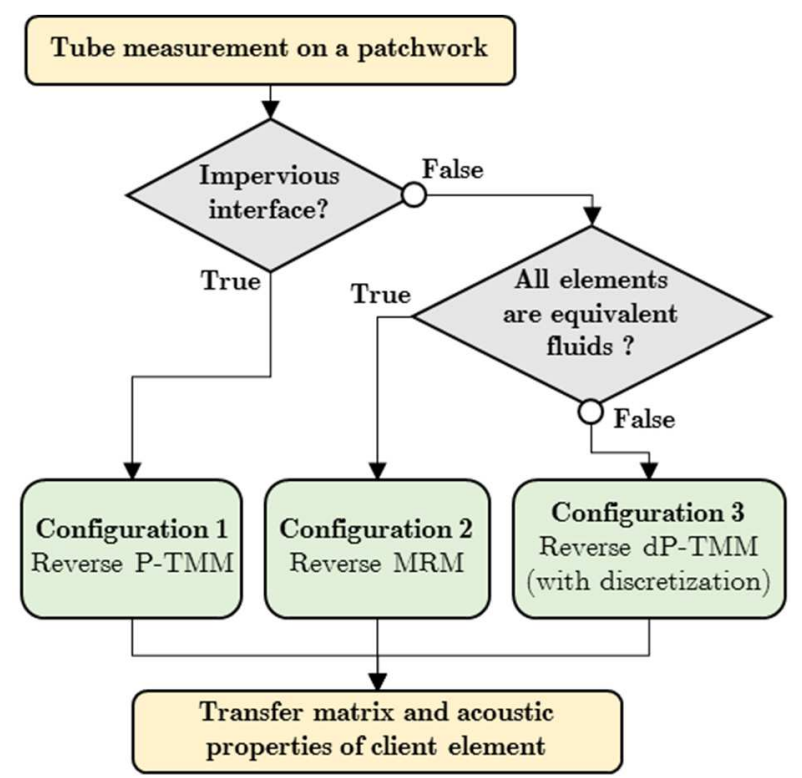

Figure 2 : Flowchart of the proposed method and choice of the characterization approach of the client element depending on the configuration type of the two-element (host-client) patchwork.

The following section details the three approaches. However, before detailing the methods underlying these approaches, it should be emphasized that they can be applied provided that the acoustic wavelength is much greater than the lateral size of the patchwork. This is also discussed in reference. $^{17}$

\subsection{Reverse parallel transfer matrix method}

\subsubsection{Parallel Transfer Matrix Method (P-TMM)}

The P-TMM is a method to predict, under assumptions, the sound absorption and the sound transmission loss of multilayered materials stacked in parallel in the form of a patchwork. This approach assumes that there is no interaction between lateral elements and each element can be represented by a 2-by-2 transfer matrix. The details on the approach are given by Verdière et al. in references. ${ }^{17-19}$ The 2-by-2 transfer matrix of the patchwork is defined as: 


$$
\left\{\begin{array}{l}
P \\
U
\end{array}\right\}=\mathbf{T}_{p}\left\{\begin{array}{l}
P^{\prime} \\
U^{\prime}
\end{array}\right\}=\left[\begin{array}{ll}
t_{p, 11} & t_{p, 12} \\
t_{p, 21} & t_{p, 22}
\end{array}\right]\left\{\begin{array}{c}
P^{\prime} \\
U^{\prime}
\end{array}\right\},
$$

where $P$ and $U$ are the acoustic pressure and velocity at the input surface, $P$ ' and $U^{\prime}$ are the acoustic pressure and velocity at the output surface, $t_{p, m n}$ are the coefficients of $\mathbf{T}_{p}$, and subscript $p$ indicates a property related to the patchwork.

In the case of the patchwork shown in Figure 1, with two elements in parallel (subscript $c$ for client, and subscript $h$ for host), the coefficients of $\mathbf{T}_{p}$ are given by (eq.10 of reference [17]) :

$$
\begin{aligned}
& t_{p, 11}=\frac{1}{D}\left(r_{c} \frac{t_{c, 11}}{t_{c, 12}}+r_{h} \frac{t_{h, 11}}{t_{h, 12}}\right) \\
& t_{p, 12}=\frac{1}{D} \\
& t_{p, 21}=\frac{1}{D}\left(r_{c} \frac{t_{c, 11}}{t_{c, 12}}+r_{h} \frac{t_{h, 11}}{t_{h, 12}}\right)\left(r_{c} \frac{t_{c, 22}}{t_{c, 12}}+r_{h} \frac{t_{h, 22}}{t_{h, 12}}\right)-\left(\frac{r_{c}}{t_{c, 12}} \operatorname{det}\left(\mathbf{T}_{\mathbf{c}}\right)+\frac{r_{h}}{t_{h, 12}} \operatorname{det}\left(\mathbf{T}_{\mathbf{h}}\right)\right) \\
& t_{p, 22}=\frac{1}{D}\left(r_{c} \frac{t_{c, 22}}{t_{c, 12}}+r_{h} \frac{t_{h, 22}}{t_{h, 12}}\right)
\end{aligned}
$$

where $D=r_{c} / t_{c, 12}+r_{h} / t_{h, 12}, t_{c, m n}$ and $t_{h, m n}$ are the coefficients of the 2-by-2 transfer matrix $\mathbf{T}_{c}$ and $\mathbf{T}_{h}$ of the client and host elements, respectively, and $r_{h}=1-r_{c}$, where $r_{c}$ is the surface area ratio (SAR) of the client element in the patchwork discussed in Section 2.1. The transfer matrix $\mathbf{T}_{c}$ and $\mathbf{T}_{h}$ can be obtained by simulation or by measurements. Examples are given in the rest of the article (equations 9 and 15). 


\section{T. Dupont et al.}

\subsubsection{Proposed method-Reverse P-TMM}

As explained above, the client element is the element to be characterized. Its properties are unknown. On the other hand, the properties of the host element are known a priori from measurements or from a theoretical model. It can be represented by the 2-by-2 transfer matrix $\mathbf{T}_{h}$. The elements are stacked in parallel. Assuming that the transfer matrix $\mathbf{T}_{p}$ of the patchwork is measured in an acoustic tube, one can retrieve from Eq. (2) the transfer matrix $\mathbf{T}_{c}$ of the client element :

$\mathbf{T}_{c}=\frac{1}{\Delta}\left[\frac{\Delta}{r_{c}}\left[\begin{array}{cc}t_{p, 11}+(\Delta-1) t_{h, 11} & r_{c} t_{p, 12} \\ \left.t_{h, 12} \operatorname{det}\left(\mathbf{T}_{h}\right)-\frac{1}{t_{p, 12}} \operatorname{det}\left(\mathbf{T}_{p}\right)+\frac{K}{t_{p, 12} \Delta}\right] & t_{p, 22}+(\Delta-1) t_{h, 22}\end{array}\right]\right.$,

where $K=\left(t_{p, 11}+(\Delta-1) t_{h, 11}\right)\left(t_{p, 22}+(\Delta-1) t_{h, 22}\right), \Delta=1-r_{h} t_{p, 12} / t_{h, 12}$, and $t_{p, m n}$ and $t_{h, m n}$ are the known coefficients of $\mathbf{T}_{p}$ and $\mathbf{T}_{h}$. Note that if the reciprocity principle hold, then the determinant of the transfer matrix will be equal to 1 (i.e., $\operatorname{det}\left(\mathbf{T}_{c}\right)=1$ ), and the coefficient $t_{c, 21}$ of $\mathbf{T}_{c}$ will reduce to $t_{c, 21}=\left(K-\Delta^{2}\right) / r_{c} t_{p, 12} \Delta$. Here, the principle of reciprocity is not imposed on the system for the sake of generality.

It is worth mentioning that in configuration 1, the two elements of the patchwork are separated from each other; they form two individual parallel cells. In this sense, this method applies to locally reacting patchworks. For this configuration 1, the procedure for characterizing the client element and the underlying method is shown schematically in Figure 3(a). 
T. Dupont et al.

a) Configuration 1 (impervious host-client interface)

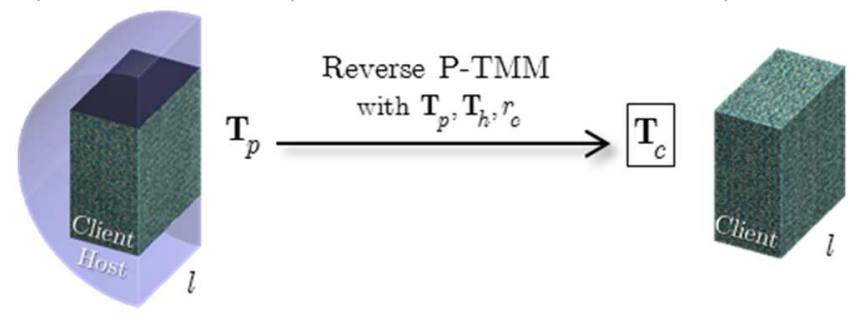

b) Configuration 2 (pervious host-client interface)

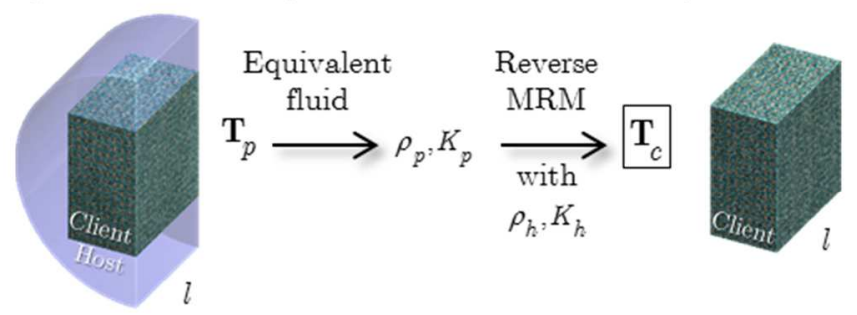

C) Configuration 3 (pervious host-client interface)

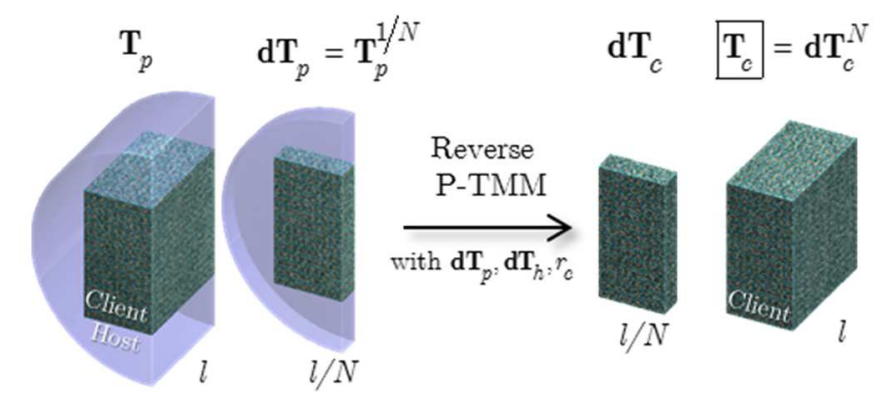

Figure 3 : Schematic of the patchwork configurations and underlying methods to retrieve the acoustic properties and transfer matrix of the Client element. (a) Configuration 1: patchwork with a rigid and impervious interface between the host and client elements. (b) Configuration 2: patchwork with a pervious interface between the two elements, where the client and host elements have to be identified as equivalent fluids. (c) Configuration 3: same as configuration 2 except the client and/or host elements have not to be identified as equivalent fluids. 
T. Dupont et al.

\subsection{Reverse Mixing Rule Method}

\subsubsection{Mixing Rule Method (MRM)}

The Mixing Rule Method ${ }^{20-23}$ (MRM) is based on the homogenization approach. In this approach, the elements of the patchwork have to be identified as equivalent fluids. Olny and Boutin ${ }^{20,21}$ have proposed an acoustic model for the double porosity media. This model takes into account the pressure diffusion effect. Based on this approach, Gourdon and Seppi ${ }^{22}$ have proposed an extension to model the acoustics of porous material with a specific porous inclusion. Chevillotte et al. ${ }^{23}$ proposed a composite model to take into account the shape of the inclusion and the end-correction for materials with high permeability contrast. It is assumed here that the two elements are macroscopically homogeneous along the thickness. Without shape effect, the relationship between the equivalent parameters of the patchwork (the dynamic density $\rho_{p}$ and the dynamic bulk modulus $\left.K_{p}\right)$ and the equivalent parameters of the two elements are given by :

$\frac{1}{\rho_{p}}=\frac{r_{c}}{\rho_{c}}+\frac{1-r_{c}}{\rho_{h}}$,

and

$$
\frac{1}{K_{p}}=F_{c} \frac{r_{c}}{K_{c}}+F_{h} \frac{1-r_{c}}{K_{h}},
$$

where $\left(\rho_{c}, K_{c}\right)$ and $\left(\rho_{h}, K_{h}\right)$ are respectively the dynamic density and the dynamic bulk modulus of the client and host elements, $F_{c}$ and $F_{h}$ are the weighting diffusion functions from host element to client element and from client element to host element, respectively. 


\section{T. Dupont et al.}

\subsubsection{Proposed method-Reverse MRM}

If no pressure diffusion is assumed (that means the surface area ratio of the client element $r_{c}$ is high and/or the permeability contrast between host and client elements is not too high), $F_{c}$ and $F_{h}$ is equal to unity. Under this assumption, $\rho_{p}$ and $K_{p}$ can be deduced from the transfer matrix of the patchwork $\mathbf{T}_{p}$ which is measured in the acoustic tube. If the host element parameters $\left(\rho_{h}\right.$ and $\left.K_{h}\right)$ are known, the dynamic density $\rho_{c}$ and the dynamic bulk modulus $K_{c}$ of the client element are simply given by :

$\rho_{c}=\left(\frac{1}{r_{c} \rho_{p}}-\frac{1-r_{c}}{r_{c} \rho_{h}}\right)^{-1}$,

and

$$
K_{c}=\left(\frac{1}{r_{c} K_{p}}-\frac{1-r_{c}}{r_{c} K_{h}}\right)^{-1}
$$

From the latter two properties, the equivalent phase velocity, the equivalent wave number, and the equivalent characteristic impedance of the client element are respectively given by :

$c_{c}=\sqrt{\frac{K_{c}}{\rho_{c}}}, \quad k_{c}=\omega \sqrt{\frac{\rho_{c}}{K_{c}}}, \quad Z_{c}=\sqrt{K_{c} \rho_{c}}$.

where $\omega$ is the angular frequency.

Finally, the transfer matrix of the client element alone, for a thickness $l$, is given by: 


\section{T. Dupont et al.}

$$
\mathbf{T}_{c}=\left[\begin{array}{cc}
\cos \left(k_{c} l\right) & j Z_{c} \sin \left(k_{c} l\right) \\
\frac{j}{Z_{c}} \sin \left(k_{c} l\right) & \cos \left(k_{c} l\right)
\end{array}\right],
$$

It is worth mentioning that it is possible to take into account the pressure diffusion effect and/or the shape effect with the help of Chevillotte et al. ${ }^{23}$ work; however for the sake of clarity only the case without diffusion is presented in this paper. For this configuration 2, the procedure for characterizing the client element and the underlying method is shown schematically in Figure 3(b).

\subsection{Reverse discretized parallel transfer matrix method}

\subsubsection{Discretized Parallel Transfer Matrix Method (dP-TMM)}

In Section 2.3, the P-TMM approach was developed for a patchwork with independent lateral elements (patchwork with impervious lateral interface between host and client elements). It has been demonstrated that for thin patchworks and/or at low frequencies, the P-TMM is similar (in a first order of approximation) to the MRM. ${ }^{27}$ Based on this observation, Verdière et al. ${ }^{19}$ proposed an extension to the P-TMM for the patchworks with lateral interactions between the lateral elements. It is proposed to subdivide in a virtual way the parallel assembly following its thickness to obtain a very thin virtual slide. This method relies on a homogeneization criterion applied on the thickness of the virtual element. This approach is called the discretized P-TMM, or AP-TMM. It can be noticed that there is no equivalent fluid assumption for the patchwork elements. This method needs only the 2-by-2 transfer matrices of the patchwork elements. Therefore, the equivalent fluid assumption for the patchwork is not required. Such a patchwork can be found in reference, ${ }^{33}$ where a patchwork of Helmholtz resonators embedded in a poroelastic foam is characterized experimentally and numerically by a 2-by-2 transfer matrix. This method can thus deal with patchworks composed of 


\section{T. Dupont et al.}

non-reciprocal elements or non-homogeneous elements according to the thickness; in the section IV of this paper (experimental part) the proposed method is tested on a non-homogeneous element (an shoddy felt).

\subsubsection{Proposed method-Reverse dP-TMM}

It is proposed to apply the AP-TMM in a reverse way. As noted above, this approach can deal with patchwork for which the lateral interactions can occur between the two elements and for which the equivalent fluid assumption is not required for the patchwork element. The experimental procedure for this configuration is presented as follows. First the global transfer matrix $\mathbf{T}_{p}$ of the patchwork is measured for a given surface ratio $r_{c}$. Second, the global patchwork is virtually subdivided (following the thickness) into $N$ independent and identical sub-layers (see Figure 3(c)). The thickness of each layer is given by $l / N$. The number $N$ of the sub-layer is defined with the help of the homogenization criterion. The sub-layer transfer matrix of the patchwork $\mathbf{d} \mathbf{T}_{p}$ is calculated with $\mathbf{d} \mathbf{T}_{p}=\mathbf{T}_{p}^{1 / N}$. Third, the sub-layer transfer matrix of the client element $\mathbf{d} \mathbf{T}_{c}$ is obtained from the reverse P-TMM, Eq. (3), with $\mathbf{d T}_{p}$ and the a priori known sub-layer transfer matrix of the host

element $\mathbf{d} \mathbf{T}_{h}=\mathbf{T}_{h}^{1 / N}$. Finally, the transfer matrix of the client element is given by $\mathbf{T}_{c}=\mathbf{d} \mathbf{T}_{c}^{N}$. The characterization procedure of this third configuration is summarized in Figure 3(c).

\subsection{Acoustic properties}

Once the transfer matrix of the client element $\mathbf{T}_{c}$ is defined, the acoustic properties for the client element alone can be deduced. Then, from the coefficients $t_{c, m n}$ of $\mathbf{T}_{c}$ the normal incidence sound absorption coefficient of the rigidly backed sample is given by : 
$\alpha=1-\left|\frac{t_{c, 11}-t_{c, 21} Z_{0}}{t_{c, 11}+t_{c, 21} Z_{0}}\right|^{2}$

and the normal incidence sound transmission loss (in transmission setup in which the sample is coupled to two semi-infinite regions) is given by :

$T L=20 \log \left(\frac{1}{2}\left|t_{c, 11}+t_{c, 22}+\frac{t_{c, 12}}{Z_{0}}+t_{c, 21} Z_{0}\right|\right)$

where $Z_{0}$ is the characteristic impedance of air at the test conditions.

\subsection{Proposed mounting configurations in an acoustic tube}

Examples of the application of configuration 1 have already been presented in details in reference [24]. In the following parts of this article, we will focus on configurations 2 and 3, where the interface between the host element and the client element is pervious. We suggest one mounting setup that minimize or even eliminates the effects of the peripheral conditions on the client test sample. This setup consists in using the client sample in parallel with air. Here, the air serves as the host element and surrounds the client in the tube. This assembly is the one shown in Figure 1. The only important parameter of the assembly is the surface area ratio of the client in the patchwork. It can be noticed that to minimize the experimental error it is recommended to minimize the volume of air element as much as possible, that means maximizing the surface area ratio of the client sample $r_{c}$.

This setup is a very convenient. It can be noticed that this setup permits the testing of samples with a surface cross-section with geometries and sizes that can be different than the tube cross- 
section. Moreover, the air host is theoretically known for the room conditions prevailing during a measurement. That simplifies the application of the proposed reverse methods.

In the next two sections, numerical validations and experimental applications of the reverse methods will be performed with this mounting configuration. The two different approaches (Reverse MRM and Reverse discretized (P-TMM) will be compared to virtual measurements simulated with the finite element method (section 3), and to real sound transmission tube measurements (section 4). As it has been mentioned previously it will be assumed that no pressure diffusion takes place. That means that the surface area ratio of the client element is high and/or the high permeability contrast between host and client elements is not too high. This condition is discussed in section 6 , where two methods are proposed to check that no pressure diffusion occurs, or very little. Moreover, it is assumed that the wavelength has to be larger than the elementary patchwork, which is also the assumption behind acoustic tube measurements.

\section{Numerical part}

\subsection{Numerical validation}

For the numerical validation, a virtual acoustic tube simulated with Finite Elements (FE) is used. The objective is to characterize an acoustic material (client element) when it is placed in a host element. It is assumed that the host element is known and the client element is unknown. Both elements are described as equivalent fluids following the Johnson-Lafarge (JL) model (Equation 


\section{T. Dupont et al.}

5.50 to 5.53 in reference ${ }^{26}$ ). The rigid frame assumption is considered here. The transfer matrix of the host element $\mathbf{T}_{h}$ is given by :

$$
\mathbf{T}_{h}=\left[\begin{array}{cc}
\cos \left(k_{h} l\right) & j Z_{h} \sin \left(k_{h} l\right) \\
\frac{j}{Z_{h}} \sin \left(k_{h} l\right) & \cos \left(k_{h} l\right)
\end{array}\right],
$$

where, $k_{h}$ and $Z_{h}$ are the equivalent wave number and acoustic impedance of the host element and $l$ is the patchwork thickness (the host and the client element have same thickness).

In the following, the Reverse MRM approach will be compared to the Reverse dP-TMM approach and to the expected properties of the client element. Both inverse approaches will be powered with virtual measurements of the patchwork transfer matrix $\mathbf{T}_{p}$. To obtain this transfer matrix, the three-microphone method ${ }^{28}$ that complements the ASTM E2611 standard is simulated with a 3D acoustical FE model using COMSOL software. In the FE model, parabolic tetrahedral elements are used to mesh the different acoustic domains of the tube and convergence of the results was verified in the frequency range of interest. The two elements of the patchwork were modeled as equivalent fluids using their complex sound speed ( $c_{h}$ for the host, and $c_{c}$ for the client) and their dynamic density ( $\rho_{h}$ for the host, and $\rho_{c}$ for the client) given by the rigid-frame JL model. Note that for the host, the sound speed and density of air at standard conditions are used (i.e., $c_{h}=344.9 \mathrm{~m} / \mathrm{s}$, $\left.\rho_{h}=1.18 \mathrm{~kg} / \mathrm{m}^{3}\right)$. Plane wave at normal incidence is chosen for the acoustic excitation.

The simulated patchwork consists of an air ring at standard conditions in which is centered a cylinder of melamine foam that acts as the client. The thickness of the patchwork, and so of the two elements, is $25.4 \mathrm{~mm}$. The diameter of the simulated tube, and so of the patchwork, is $44.44 \mathrm{~mm}$. The diameter of the melamine foam is $38.2 \mathrm{~mm}$. Consequently, the surface area ratio of the 


\section{T. Dupont et al.}

melamine foam in the patchwork is $r_{c}=73.9 \%$. There is no impervious lateral interface between the two elements. The JL parameters of the client melamine foam are given as follow: the open porosity is $99 \%$, the static airflow resistivity is $12000 \mathrm{~Pa} \cdot \mathrm{s} / \mathrm{m}^{2}$, the tortuosity is 1.01 , the viscous and thermal lengths are respectively $100 \mu \mathrm{m}$ and $400 \mu \mathrm{m}$, and the bulk density is $9 \mathrm{~kg} / \mathrm{m}^{3}$ (Table 1 of reference $\left.{ }^{29}\right)$.

In a practical case, the JL parameters of the client element are a priori unknown. Here, they are only used to feed the FE model of the virtual tube measurement. The application of the two proposed Reverse MRM and dP-TMM approaches are not be fed with these parameters, only those of the air ring are used as explained in sections 2.3 and 2.4 .

The comparison on the acoustic properties (absorption and transmission) between FE results and the results of the present reverse approaches are given in Figure 4. Four results are presented on the same figure: the result of the regular configuration (full client element alone in the tube, sample with the same diameter as the tube diameter), the raw results on the patchwork (host + client) and the results of the two proposed approaches (Reverse MRM and AP-TMM) applied on the raw results. For this numerical case, one can note that the two proposed reverse approaches give the exact acoustic results of the client alone. The reverse approaches perfectly removes the effect of the lateral air ring host surrounding the melamine foam sample in the patchwork configuration. This validates the implementation of the proposed reverse approaches for the case where both elements can be identified as equivalent fluids. 
T. Dupont et al.
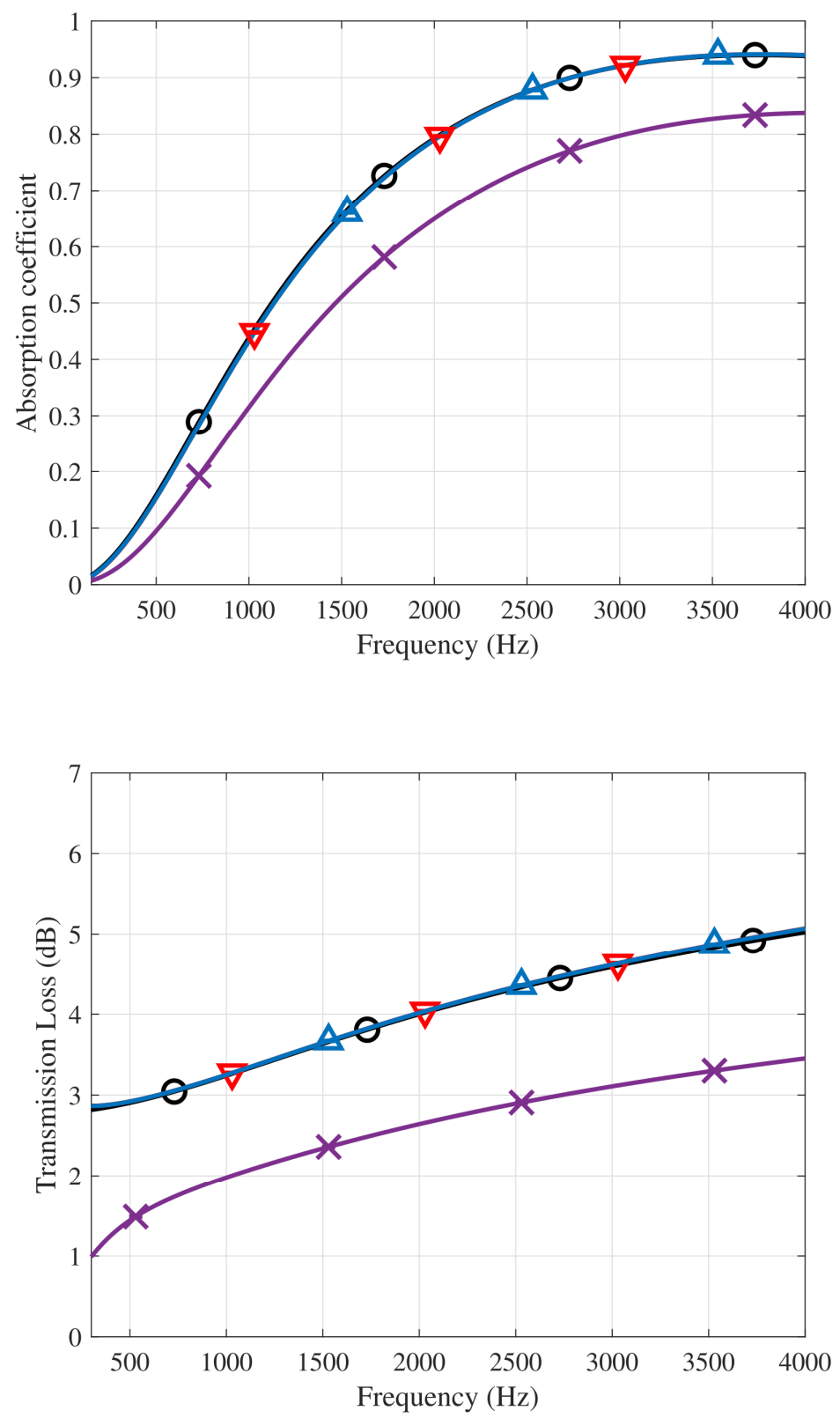

Figure 4 : Normal incidence sound absorption coefficient (sample backed by a rigid piston) and normal incidence sound transmission loss of a 25.4-mm thick sample of Melamine foam. Three approaches are presented: the direct tube measurements in regular configuration (sample alone in the simulated tube) (-๑-), direct measurement on proposed mounting configuration (the sample with 


\section{T. Dupont et al.}

$26 \%$ air host) (-X-), the results based on the proposed mounting configuration with Reverse MRM

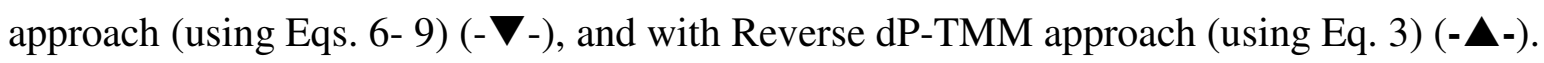

\subsection{Discussion on the effects of boundary condition and frame type}

The objective of the proposed reverse approaches is to obtain, with a finite size sample, the acoustic behavior of a sample of infinite lateral dimensions. The most appropriate lateral boundary condition in this ideal configuration is the sliding edge condition. ${ }^{8}$ However, it is unlikely that the sliding edge condition will be achieved experimentally in a tube. In reality, the actual mounting condition is unknown and is more likely to be between bonded edge and the air leak. This is why it is proposed here to force an air leak condition, using a lateral air ring as illustrated in Figure 1, to have a controlled boundary condition. With this controlled lateral boundary condition, it shall be possible to eliminate the boundary condition effects with the proposed reverse approaches.

In this subsection, we underline the effects of different lateral boundary conditions (sliding edge, air ring, bonded edge) on the normal incidence transmission loss of a sample of melamine foam in an acoustic tube. Figure 5 shows the normal incidence sound transmission loss of a 25.4-mm thick sample of melamine foam with an air leak varying from $0 \%$ (no air leak) to $2 \%$ around its edge (the percentage of air leak is equivalent to ratio $r_{c}$ described above in a 44.44-mm diameter tube). The transmission loss is numerically calculated for three different types of frame assumptions: rigid-frame (equivalent fluid model), limp-frame (equivalent fluid model), ${ }^{30}$ and elastic frame (Biot poroelastic model). ${ }^{26}$ The material parameters of the poroelastic melamine foam are given in Table 1 of reference. ${ }^{29}$ 


\section{T. Dupont et al.}

Concerning simulations with the equivalent fluid model (for rigid and limp frames), they implicitly imply laterally infinite dimensions because no frame displacement, on which sliding or bonded edge conditions are imposed, exists in this model. Concerning the simulations with the Biot model, the air leak still varies from $0 \%$ to $2 \%$. However, when air leak is $0 \%$ (no air leak), two boundary conditions are applied on the edge displacements: sliding edge (dotted line) and bonded edge (solid line). As one can observe in Figure 5, the Biot's poroelastic model, with bonded edge condition, has an acoustic behavior close to the rigid-frame model (blue area) until the first elastic resonance of the skeleton. Beyond this resonance, its acoustic behavior approaches that of the limpframe model. On the other hand, the Biot's poroelastic model with air leak (grey area), or without air leak and the sliding edge condition, behaves quite similarly to the limp-frame model (pink area) on almost all the frequency range. However, at higher frequencies, due to higher order elastic resonances, the transmission loss oscillates.

The previous results show to what extent the normal incidence measurements of a porous sample in a tube are affected by its lateral boundary conditions and the hypothesis of the frame type. In reality, a porous sample has an elastic frame and its edge displacements are difficult to control in a tube and generally unknown - somewhere between the bonded edge and air leak. A controllable mounting condition in a tube is that of the air ring (controlled air leakage). This is why the method proposed in this article takes advantage of this mounting condition, where the test sample (client) is surrounded by an air ring (host). 
T. Dupont et al.

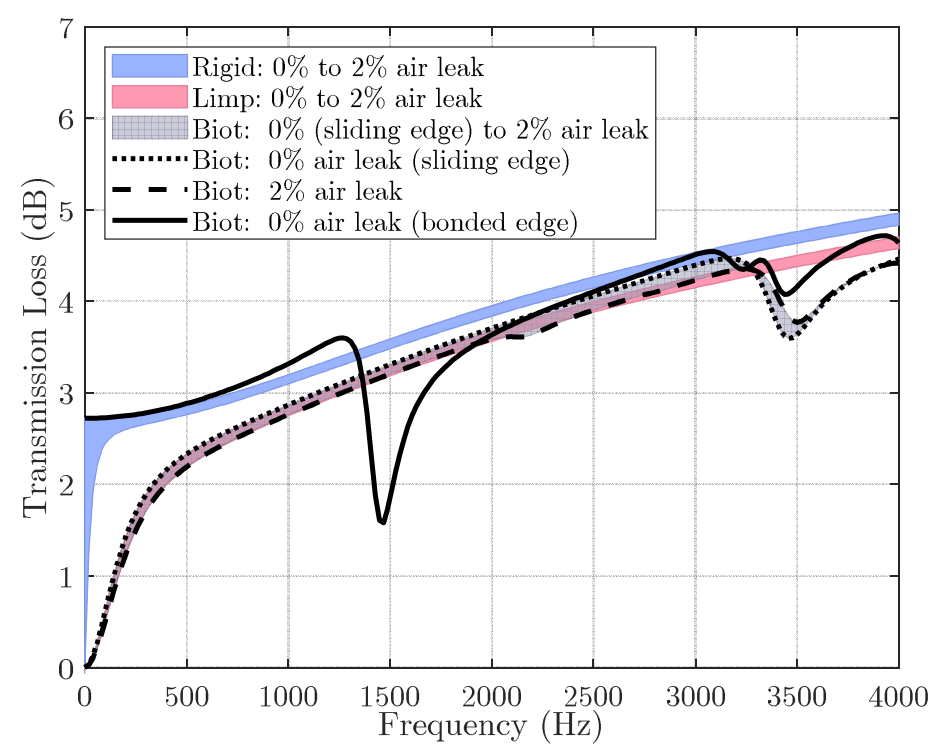

Figure 5 : Numerical predictions of the normal incidence sound transmission loss of a 25.4-mm thick sample of melamine foam in a 44.44-mm tube with an air leak varying from $0 \%$ (no leak) to $2 \%$. Results are presented for the rigid-frame (blue area) and limp-frame (pink area) assumptions (equivalent fluid model), for the elastic frame assumption (Biot model) with air leak (grey area), and for elastic frame assumption without air leak and with sliding edge condition (dotted line) and bonded edge condition (solid line).

\section{Experimental part}

\subsection{Presentation}

In this part, the reverse approaches proposed, with the air-host mounting condition, are applied to two porous client materials. The first is a melamine foam which is a homogeneous foam (see 


\section{T. Dupont et al.}

Figure 6(c)). The second is a shoddy felt which is not uniform according to its thickness (see Figure 6(d)). It is denser on one side than on the other side.

Each client material sample is tested with an air ring and rests on a support grid as shown in Figure 6(a)-(d). For this mounting configuration, the tube is in a vertical position and the client sample, with a cross-section smaller than the tube's cross-section, is placed in the center of the tube with the help of the support grid (see Figure 6(a)-6(b)). This configuration eliminates all lateral contact between the client sample and the tube's wall. However, sometimes the grid can introduce certain artefacts when applying the reverse method. Recommendations for identifying these artefacts are presented in section 6. For a better homogenization of the pressure near the input and output surfaces of the patchwork, it is recommended to subdivide the client sample into smaller elements (see Figure 6(c)-6(d)). In addition, in order to minimize the effect of pressure diffusion, it is recommended to use the highest client surface ratio $r_{c}$. These recommendations are discussed in section 6.

In the following, circular samples of $38.2 \mathrm{~mm}$ in diameter are used in a circular acoustic tube of $44.44 \mathrm{~mm}$ in diameter. In addition, all the samples are divided into four parts along the thickness (see Figure 6(c)-6(d)). The equivalent patchwork is composed of $73.9 \%$ of the client element and $26.1 \%$ of air-ring host.

The transfer matrix $\mathbf{T}_{p}$ of a patchwork is measured with an acoustic tube following standard ASTM E261 $1^{1}$ and the three-microphone and two-load method ${ }^{28}$. This technique is generally used to measure the normal incidence acoustic properties and transfer matrix of a porous sample coupled to two different air cavities backed by a rigid termination. The frequency range was chosen between $150 \mathrm{~Hz}$ to $4000 \mathrm{~Hz}$ to ensure that only plane waves exist in the region near to the microphone 
positions in a $44.44 \mathrm{~mm}$ diameter tube. Using two microphones upstream the sample and a third microphone located on the hard wall backing, the transfer matrix and the acoustic indicators (sound absorption coefficient and transmission loss) are deduced. The sound pressure excitation is random noise in the linear regime. As recommended in standard ASTM E2611 ${ }^{1}$, to be sure that the amplitude of the plane wave dominates at the microphone positions in the tube, at least one tube diameter separates the test sample to the nearest microphone. The depth of the rear air cavity is chosen to be greater than two tube diameters.

The effect of the grid needs first to be removed on the measured transfer matrix. In fact, in this configuration, the grid is in series with the patchwork. Consequently, the measured transfer matrix for the patchwork is in series with the grid. To remove the effect of the grid, the following operation is applied:

$$
\mathbf{T}_{p}=\mathbf{T}_{g}^{-1} \mathbf{T}_{p+g},
$$

where $\mathbf{T}_{g}$ is the transfer matrix of the grid, and $\mathbf{T}_{p+g}$ is the measured transfer matrix of the patchwork in series with the grid. The transfer matrix of the grid is measured with the acoustic tube, or theoretically evaluated by :

$$
\mathbf{T}_{g}=\left[\begin{array}{cc}
\cos \left(k_{g} l_{g}\right) & j Z_{g} \sin \left(k_{g} l_{g}\right) \\
\frac{j}{Z_{g}} \sin \left(k_{g} l_{g}\right) & \cos \left(k_{g} l_{g}\right)
\end{array}\right],
$$

where $l_{g}$ is the thickness of the grid, and $k_{g}$ and $Z_{g}$ are respectively the equivalent wave number and equivalent impedance of the grid. These equivalent properties can be defined by an equivalent fluid approach as described in Section 4.6 of reference. ${ }^{26}$ 
T. Dupont et al.

Since the host element is air, its transfer matrix is theoretically given by :

$\mathbf{T}_{h} \rightarrow \mathbf{T}_{h}^{a i r}=\left[\begin{array}{lc}\cos \left(k_{0} l\right) & j Z_{0} \sin \left(k_{0} l\right) \\ \frac{j}{Z_{0}} \sin \left(k_{0} l\right) & \cos \left(k_{0} l\right)\end{array}\right]$

where $k_{0}$ and $Z_{0}$ are the wave number and acoustic impedance of the air at the test conditions and $j=\sqrt{-1}$.

With the help of $\mathbf{T}_{p}, \mathbf{T}_{h}^{\text {air }}$, and $r_{c}$, the transfer matrix of the client element $\mathbf{T}_{c}$ can be defined either by Reverse dP-TMM approach (section II-D with Eq. 3) or by Reverse MRM approach (section II-C with Eqs. 6-9).

During measurements, the repeatability errors come mainly from the cutting process of the sample and its position in the tube: special attention was therefore paid to the cutting process and the positioning. For each client material, at least five measurements on five different samples are used to calculate the mean value and standard deviation of the acoustic indicators. The results are presented with the mean value $\bar{x}$ and the expanded uncertainty with a coverage factor $^{31} h=2$ : $x=\bar{x} \pm h \sigma_{x}$, with $\sigma_{x}$ the standard deviation.

In the following, the results of the proposed reverse approaches are compared with two direct measurements. The first one is the direct measurement on the sample alone (regular mounting configuration). In this case, the sample has the same diameter as the tube and its lateral surface is in contact with the wall of the tube. The second direct measurement is on the client sample with the air ring host. This is the mounting configuration proposed in Figure 6. In this case, the sample is not in contact with the wall of the tube. 
T. Dupont et al.
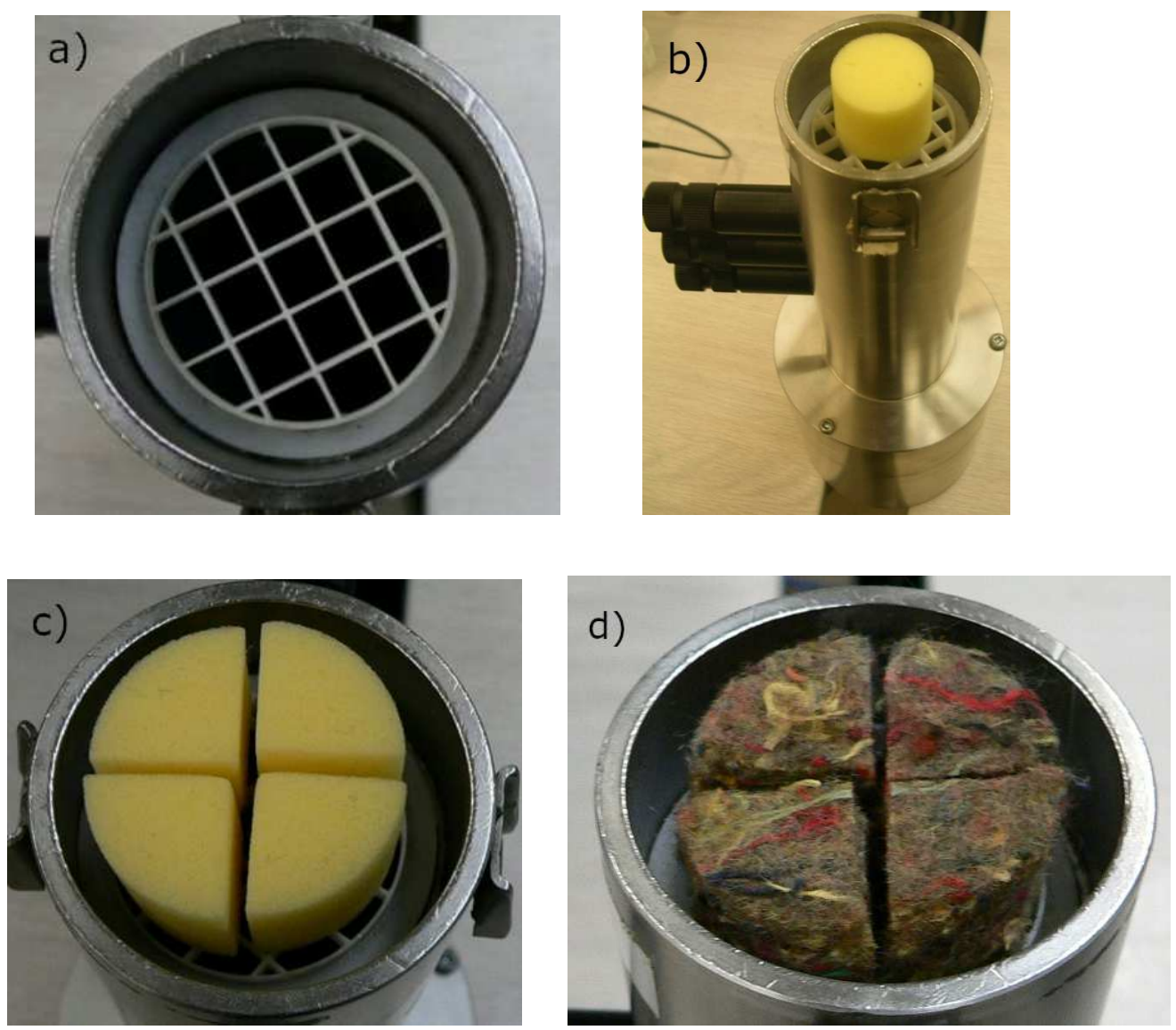

Figure 6 : Photos of the proposed mounting configurations. The client element is tested with air host element and a grid (see photo (a)). The tube is in vertical position (see photo (b)). It is recommended to maximize the air element surface ratio and to cut the sample in many smaller lateral size elements; examples are given for a 25.4-mm thick cylinder of a melamine foam (photo (c)) and for a 18-mm thick cylinder of shoddy felt (photo (d)). 


\section{T. Dupont et al.}

\subsection{Homogeneous porous samples}

The first client element tested in the acoustic tube is a $25.4-\mathrm{mm}$ thick cylinder of a homogeneous melamine foam subdivided into 4 elements as shown in Figure 6(c). The static airflow resistivity of this melamine foam is $10050 \mathrm{~Pa} . \mathrm{s} / \mathrm{m}^{2}$. Figure 7 presents the acoustic indicators directly measured on the two mounting configurations: regular configuration (sample alone with the same diameter than the tube and with unknown boundary condition) and proposed patchwork configuration (client sample with $26.1 \%$ air host).

For the regular configuration (-O-), by comparing its transmission loss with that of Fig. 5, it can be assumed that the constraining effect of the wall of the tube on the sample is very close to that of a bonded edge condition. This is not always the case since the contact depends strongly on the quality of the sample (its circularity and diameter compared to those of the tube) and on the friction coefficient between the sample and the tube.

For the proposed patchwork configuration $(-\times-)$, one notes that adding the air host changes the acoustic indicators; however this time, the boundary condition (air-porous interface) is very well known. By applying the reverse approaches to this measurement with the host air, we eliminate the effect of the host air and we recover the indicators of the client alone, without the constraining effects of the tube wall.

Figure 7 presents these indicators deduced from the two proposed reverse approaches (Reverse

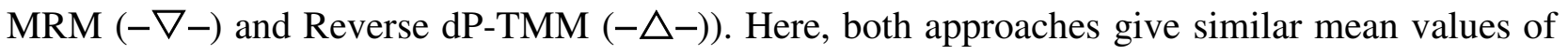
the acoustic indicators. In addition, the first frame resonance has disappeared and the behavior tends, as expected in Sec. 3.2, to a limp-frame behavior. In other words, the air-ring condition tends to 
T. Dupont et al.

modify the rigid frame behavior of a sample into a limp frame behavior over a wide frequency bandwidth.
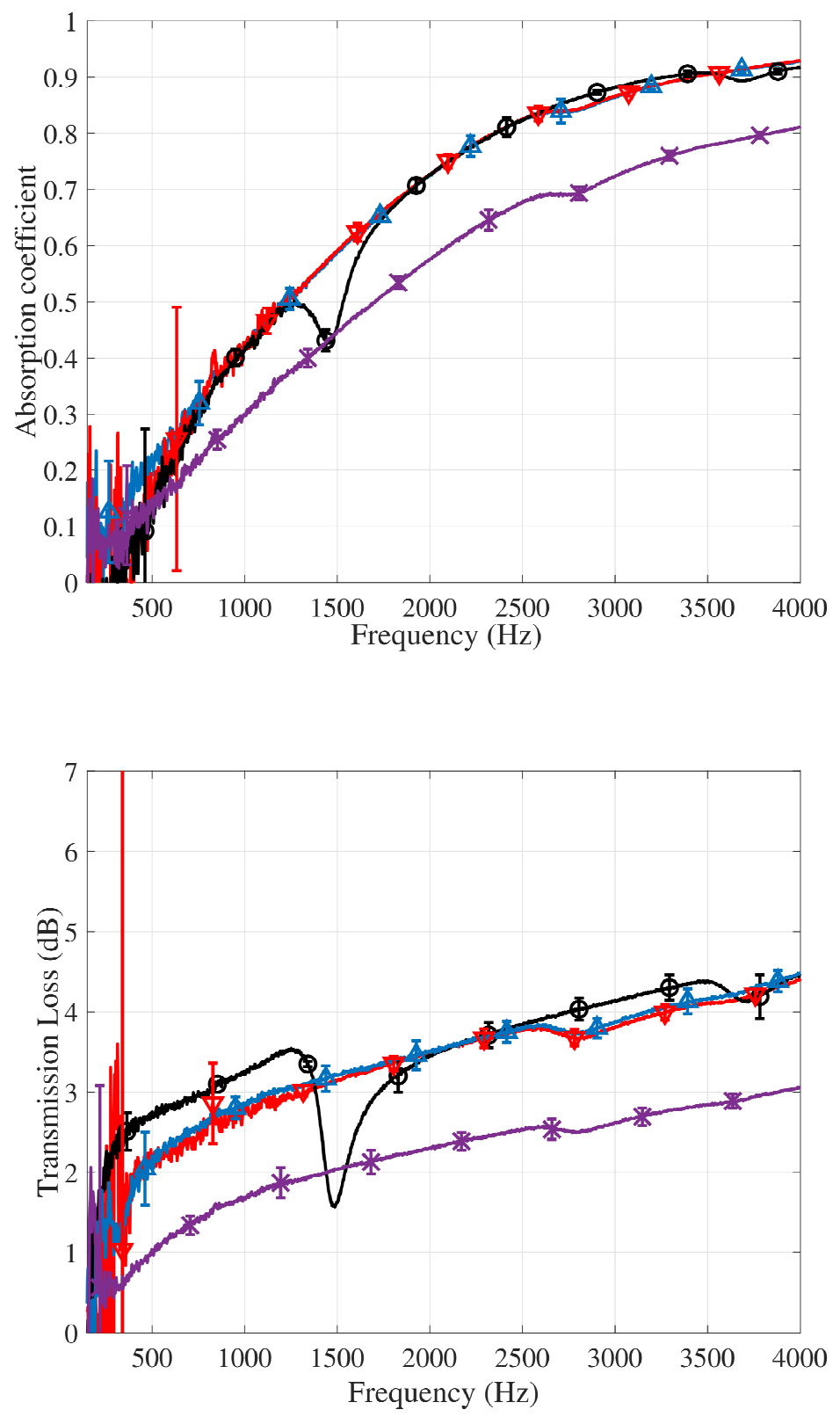

Figure 7 : Normal incidence sound absorption coefficient (rigid backing condition) and normal incidence sound transmission loss of a 25.4-mm thick melamine foam. Direct measurement on the regular configuration (sample 


\section{T. Dupont et al.}

alone with no air host) (-O-); direct measurement on the sample with $26 \%$ air host (-X-); Reverse MRM approach (using Eqs. 6-10) (- $\nabla$-), and Reverse dP-TMM approach (using Eq. 3) (- $\triangle$-).

\subsection{Non-homogeneous porous samples}

The second client element is a 18-mm thick shoddy felt (see Figure 6(d)). The static airflow resistivity of this felt is $20500 \mathrm{~Pa} . \mathrm{s} / \mathrm{m}^{2}$. Contrary to the melamine foam, this felt is not uniform according to its thickness. It is denser on one side than the other. Consequently, the coefficients $t_{\mathrm{c}, 11}$

and $t_{\mathrm{c}, 22}$ of its transfer matrix are expected to be not equal. ${ }^{1}$ In addition, the cutting process produced irregular contours from sample to sample. This implies a less good control of the mounting conditions in the regular configuration from one sample to another in the tube.

This non-homogeneous material cannot be theoretically identified as an equivalent fluid. To use the Reverse MRM approach, it is necessary to consider the material as an equivalent fluid. The advantage to use the Reverse dP-TMM approach is that the measured transfer matrix of the patchwork can be used directly to define the transfer matrix of the client element (there is no equivalent fluid assumption). As expected, Figure 8 shows that for a non-uniform material according to its thickness, only the Reverse dP-TMM approach gives valid results when compared to regular configuration. Again, the reverse approach removes the first elastic resonance which is very sensitive to boundary condition in a regular mounting configuration. 
T. Dupont et al.
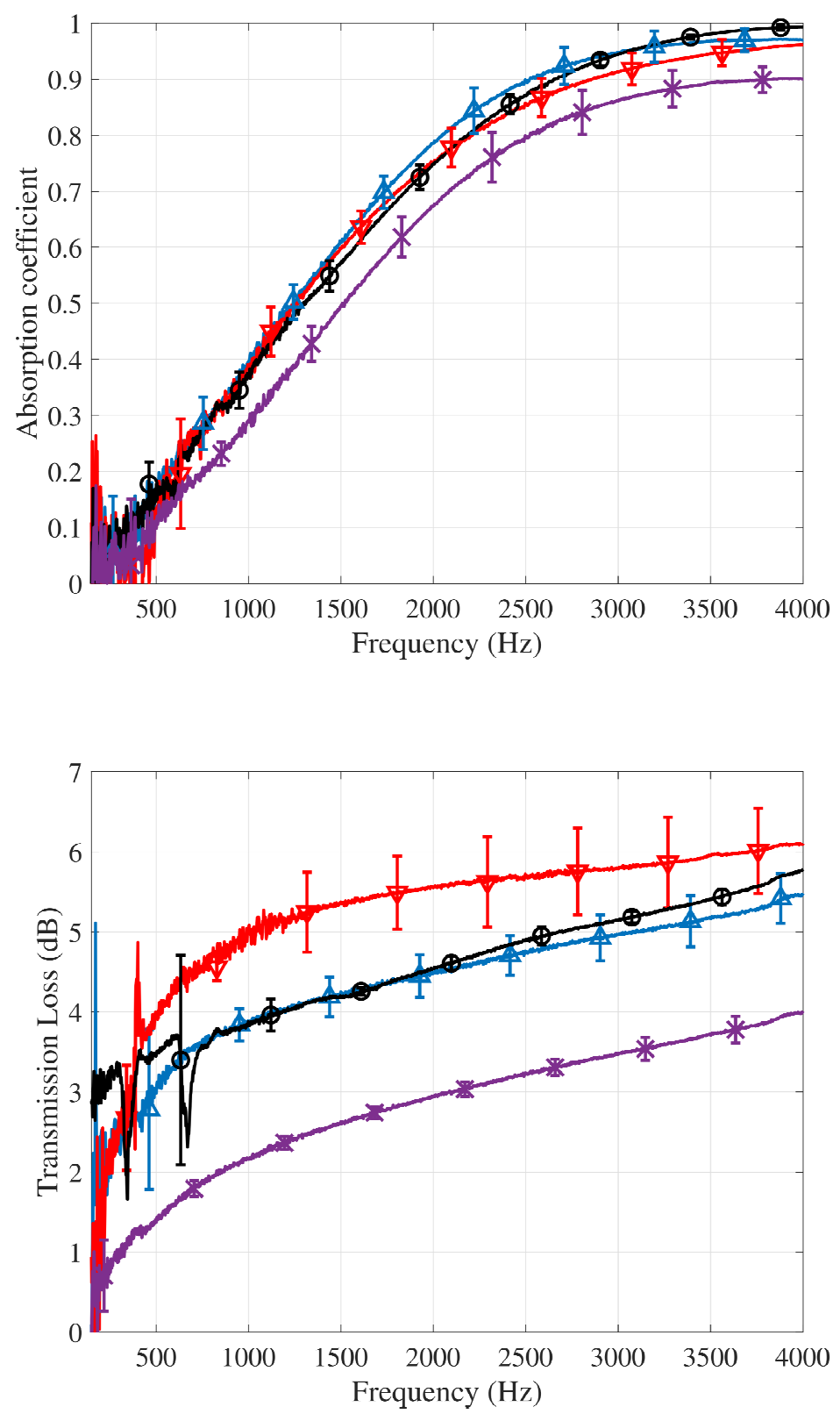

Figure 8 : Normal incidence sound absorption coefficient (rigid backing condition) and normal incidence sound transmission loss of a 18 -mm thick felt which is not uniform according to its thickness. Direct measurement on the regular configuration (sample alone with no air host) (-O-); direct measurement on the sample with $26 \%$ air host (-×-); Reverse MRM approach (using Eqs. 6-10) (- $\nabla-$ ), and Reverse dP-TMM approach (using Eq. 3) (- $\triangle$-). 


\section{T. Dupont et al.}

\section{Discussion}

It can be noticed that at the first order of approximation, the MRM approach and dP-TMM approach give exactly the same results for low frequencies and/or for thin samples. ${ }^{27}$ However, the MRM approach requires that the two elements of the patchwork can be considered as equivalent fluids (while the P-TMM approach needs only a 2-by-2 transfer matrix of the patchwork). In the MRM approach, in order to identify the equivalent parameters of the patchwork $\rho_{p}$ and $K_{p}$, the inverse cosine (or inverse sine) function has to be applied on the coefficient of the transfer matrix of the patchwork (which is measured in the acoustic tube). Depending on the experimental conditions and on material properties, these functions can cause phase errors, and this can induce important errors on the calculation of the transfer matrix of the client element. Thus, for nonhomogeneous or non-reciprocity materials, or materials which cannot be modeled as equivalent fluids, it is recommended to use the Reverse dP-TMM approach.

Concerning the Reverse dP-TMM approach, the number $N$ of the virtual sub-layers depends on the equivalent phase velocities in the two elements, at the maximum frequency of the study (here $4000 \mathrm{~Hz}$ ), and on the patchwork thickness $l . N$ must be a value much larger than the proposed criterion. Since the phase velocity of one (or two) element(s) is, a priori, unknown, a convergence study on acoustic parameters could be used to precisely define $N$. For example, for the studied samples, it was found that for $N \geq 3$ ensures convergence of the acoustic parameters. Since, computation time is not an issue using a large value of $N$, one can use a value of $N=10$, or even much larger, by default. 


\section{T. Dupont et al.}

\section{Precautions for use}

When using the proposed reverse method, precautions should be taken with caution. The first concerns the artifacts introduced by the grid - if a support grid is used, and the second the possibility of pressure diffusion from the air ring host and the client element - if the client element is a porous medium.

As shown in Figure 6 a grid would be used to eliminate edge constraints, subdivide client material, or help support a thin client material that cannot stand by itself in the configuration shown in Figure 1. In such a mounting condition, the acoustical effect of the grid must be removed using Eqs. (13) and (14). This works fine if the host element is motionless or rigid. However, if the frame of the host element is elastic (ex. poroelastic foam), the grid constraints the axial motion on the rear face of the host. In some situations, when the coupling between acoustic and elastic compressional waves is strong enough, this will add artefacts in the results of the proposed reverse method, when compared to an ideal measurements. This is because the ideal situation assumes (as per ASTM E2611) that the host separates two air media (transmission problem) without constraints on the axial movement, whereas in measurements with a grid there is. Consequently, the transfer matrix recovered by the proposed method hides a parasitic elastic contribution of the frame. For a poroelastic material, this parasitic elastic contribution is more important below the visco-inertial

decoupling frequency ${ }^{34}$ given by $f_{d}=\phi^{2} \sigma / 2 \pi \rho_{1}$, where $\phi, \sigma$, and $\rho_{1}$ are the open porosity, static airflow resistivity and bulk density of the material. If these parameters are known, one can easily evaluate below what frequency this parasitic contribution, and therefore the grid effect, will mainly add artefacts to the reverse method. For the melamine foam and the shoddy felt studied previously, the decoupling frequencies are lower than $175 \mathrm{~Hz}$ and $40 \mathrm{~Hz}$, respectively. This is why none of these 


\section{T. Dupont et al.}

artefacts occurred in the application of the method. When the material properties are not known, the experimenter can check if a large drop is observed in the TL of the direct measurement on the patchwork (host and client). If so, it may be due to compressive elastic resonance due to the use of the support grid. In this case, care should be taken when interpreting the results.

The proposed method assumes that the pressure diffusion is absent or negligible. Pressure diffusion may occur when the host is air and the client material is an open-cell porous material. To ensure that no pressure diffusion occurs over the studied frequency bandwidth, it is recommended to use the largest possible client element surface ratio $r_{c}$. If the resistivity of the client material is known, it is recommended to use a surface ratio $r_{c}>r_{c m i n i}$, where $r_{c \min }$ is the minimal value of the client element surface ratio from which no pressure diffusion will occur on the studied frequency bandwidth. $r_{c \min }$ depends on the client element static airflow resistivity and is defined from a numerical parametric study based on the average relative difference (ARD). ${ }^{32}$ Here, ARD is calculated based on the absorption coefficient found with the proposed reverse method and the theoretically expected one (full sample with sliding edge). Consequently, for the parametric study, the proposed reverse method is successively applied on a porous client with a resistivity varying from 10000 to $1600000 \mathrm{~Pa} \cdot \mathrm{s} / \mathrm{m}^{2}$, and a decreasing surface client ratio $r_{c}$. For each resistivity, the process stops when the reverse method results in a ARD $=6 \%$. This yields the values of $r_{c m i n}$ for the tested resistivities. These values are reported in Table I. This table can be used as a guideline to select the surface ratio to use with the method.

If the resistivity of the client material is unknown, it is recommended to test two samples of different diameters (or with two different cross-section geometries) and compare the corresponding deduced absorption coefficient curves. The average relative difference can be calculated with the 


\section{T. Dupont et al.}

two deduced absorption coefficients. If a small difference is found (eg ARD $<6 \%$ ), the pressure diffusion can be considered negligible.

Table I: Recommended minimal values of client element surface area $r_{c m i n}$ for a frequency bandwidth [50, 4400] Hz.

\section{Conclusion}

Based on the P-TMM approach or on MRM approach applied in reverse way to the parallel assembly of patchworks, a simple method to measure the acoustic indicators of a material when it is tested in a tube with parallel host element has been proposed. Three different configurations have been considered: the configurations characterized by the patchworks with and without a rigid and impervious interface between the two parallel elements (that means considering with or without lateral interactions between the elements), and the configurations with and without equivalent fluid assumptions for the elements have to be distinguished. For each configuration, a specific approach have been proposed.

A very convenient mounting setup has been proposed in which the client element is tested with a controlled air ring host element. Numerical (virtual tube) and experimental (acoustic tube) 
validations have been presented. The MRM approach and the AP-TMM approach are applied in a reverse way for different acoustic materials. If the material is homogeneous and can be considered as an equivalent fluid, the two approaches give the same results, and the comparisons with the regular configuration (full sample alone in the tube) are good. However, for non-homogeneous and/or non-reciprocal materials, or for materials which cannot be modelled as equivalent fluids, it was shown that only the dP-TMM approach yields good results. Indeed the Reverse dP-TMM approach needs only to characterize the 2-by-2 transfer matrix of the patchwork (client + air-ring host) by an experimental tube method (ex.: ASTM E2611), without any assumption on the material or system this transfer matrix represents.

On the basis of this global approach, it should be recalled that the reference [25] is complementary to this article by proposing four configurations / mounting methods which make it possible to better control the effects of the lateral boundary conditions between the sample and the acoustic tube.

\section{Acknowledgements}

This work was supported by the Natural Sciences and Engineering Research Council of Canada (NSERC) and by the Region Bourgogne-Franche Comté (France).

\section{Author's contributions}

All authors contributed equally to this work. 


\section{Data Availability Statement}

The data that support the findings of this study are available from the corresponding author upon reasonable request. References for all other datasets are given in the text.

\section{References}

1 ASTM International. E2611-19 Standard Test Method for Normal Incidence Determination of Porous Material Acoustical Properties Based on the Transfer Matrix Method. West Conshohocken, PA; ASTM International, 2019. doi: https://doi.org/10.1520/E2611-19

2 ISO 10534-2. "Acoustics-Determination of sound absorption coefficient and impedance in impedance tubes. Part 2: Transfer-function method," International Organization for Standardization, Geneva, Switzerland 1998.

3 ASTM International. E1050-19 Standard Test Method for Impedance and Absorption of Acoustical Materials Using a Tube, Two Microphones and a Digital Frequency Analysis System. West Conshohocken, PA; ASTM International, 2019. doi: https://doi.org/10.1520/E1050-19

4 R. J. Donato, “Model experiments on surface waves,'” J. Acoust. Soc. Am. 63, 700-703 (1978).

5 T.E. Vigran, L. Kelders, W. Lauriks, P. Leclaire, T.F. Johansen, "Prediction and measurements of the influence of boundary conditions in a standing wave tube". Acta Acustica united with Acustica, 83, 419-23 (1997). 
${ }_{6}$ B. H. Song, J. S. Bolton, and Y. J. Kang, "Effect of circumferential edge constraint on the acoustical properties of glass fiber materials," J. Acoust. Soc. Am. 110, 2902-2916 (2001).

7 B. H. Song and J. S. Bolton, "Investigation of the vibrational modes of edge-constrained fibrous samples placed in a standing wave tube," J. Acoust. Soc. Am. 113, 1833-1849 (2003).

8 D. Pilon, R. Panneton, and F. Sgard, "Behavioral criterion quantifying the edge-constrained effects on foams in the standing wave tube," J. Acoust. Soc. Am. 114, 1980-1987 (2003).

9 H.-S. Tsay and F.-H. Yeh, "The influence of circumferential edge constraint on the acoustical properties of open-cell polyurethane foam samples”, J. Acoust. Soc. Am. 119, 2804 (2006).

${ }^{10}$ M. Schwartz and E. J. Gohmann, "Influence of Surface Coatings on Impedance and Absorption of Urethane Foams", J. Acoust. Soc. Am. 34, 502 (1962).

${ }^{11}$ J.-F. Allard and P. Delage, "Free field measurements of absorption coefficients on square panels of absorbing materials,', J. Sound Vib. 101, 161-170 (1985).

${ }^{12}$ A. Cummings, "Impedance tube measurements on porous media: The effects of air-gaps around the sample”, J. Sound Vib., Volume 151, Issue 1, 22, 63-75 (1991).

${ }_{13}$ X. Olny and C. Boutin, “Acoustic wave propagation in double porosity media,' J. Acoust. Soc. Am. 114, 73-89 (2003).

${ }^{14}$ D. Pilon, R. Panneton, and F. Sgard, 'Behavioral criterion quantifying the effects of circumferential air gaps on porous materials in the standing wave tube', J. Acoust. Soc. Am. Volume 116, Issue 1, 344-356 (2004). 
${ }^{15}$ F. C. Sgard, X. Olny, N. Atalla, F. Castel, "On the use of perforations to improve the sound absorption of porous materials". Appl. Acoust., 66, 625-51 (2005).

${ }_{16}$ T. Dupont, P. Leclaire, O. Sicot, X. L. Gong and R. Panneton, “Acoustic properties of airsaturated porous materials containing dead-end porosity”, J. App. Phys. 110, 09490313 pp (2011).

${ }^{17}$ K.Verdière, R. Panneton, S. Elkoun, T. Dupont and P. Leclaire, "Transfer matrix method applied to the parallel assembly of sound absorbing materials", J. Acoust. Soc. Am, Volume 134, Issue 6, 4648 (2013).

${ }^{18}$ K.Verdière, R. Panneton, S. Elkoun, T. Dupont and P. Leclaire, "Comparison between parallel transfer matrix method and admittance sum method", J. Acoust. Soc. Am. 136, EL90 (2014).

${ }^{19}$ K.Verdière, R. Panneton, S. Elkoun, T. Dupont and P. Leclaire, "Recent highlights on the parallel transfer matrix method (PTMM). Proceeding of Symposium on the Acoustics of Poro-Elastic Materials (SAPEM), Stockholm, 16-18 December 2014.

${ }^{20}$ X. Olny, Ph.D. thesis, Institut National des sciences appliquees, Lyon, France, 1999.

${ }^{21}$ X. Olny and C. Boutin, "Acoustic wave propagation in double porosity media", J. Acoust. Soc. Am. 114, Volume 73 Issue 89 (2003).

${ }_{22}$ E. Gourdon and M. Seppi, "On the use of porous inclusions to improve the acoustical response of porous materials: Analytical model and experimental verification", Applied Acoustics, Volume 71, Issue 4, 283-298, (2010).

${ }^{23}$ F. Chevillotte, L. Jaouen, F. X. Bécot, "On the modeling of visco-thermal dissipations in heterogeneous porous media”, J. Acoust. Soc. Am, Volume 138, Issue 6 (2015). 
${ }^{24}$ T. Dupont, P. Leclaire, K.Verdière, R. Panneton and S. Elkoun. "A method for measuring the acoustic properties of a porous sample mounted in a rigid ring in acoustic tubes", proceeding 21st International Congress on Acoustics (ICA) Montreal, 2-7 June 2013.

${ }_{25}$ T. Dupont, K. Verdière, P. Leclaire, R. Panneton. "A method to control the lateral boundary condition effects in the characterization of acoustic materials in an impedance tube". Proceeding of Internoise, San Francisco, 9-12 August 2015.

${ }^{26}$ J. F. Allard and N. Atalla, Propagation of Sound in Porous Media: Modelling Sound Absorbing Materials, Wiley and Sons, Ltd, Southern Gate, Chichester, West Sussex, United Kingdom, 2009, 358 pp.

${ }^{27}$ C. J. Sacristan, T. Dupont, O. Sicot, P. Leclaire, K. Verdière, R. Panneton and X. L., Gong. "A mixture approach to the acoustic properties of a macroscopically inhomogeneous porous aluminum in the equivalent fluid approximation”, J. Acoust. Soc. Am., Volume 140, no. 4, 2847-2855 (2016).

${ }_{28}$ Y. Salissou, O. Doutres, R. Panneton, "Complement to standard method for measuring normal incidence with three microphones”, J. Acoust. Soc. Am., Volume 131, EL216 (2012).

${ }^{29}$ N. Geebelen, L. Boeckx, G. Vermeir, W. Lauriks, J.-F. Allard, O. Dazel, "Measurement of the rigidity coefficients of a melamine foam”, Acta Acustica united with Acustica, Volume 93, 783-8 (2007).

${ }^{30}$ R. Panneton, "Comments on the limp frame equivalent fluid model for porous media", J. Acoust. Soc. Am., Volume 122, no. 6, EL217 - EL222 (2007). 
31 Joint Committee for Guides in Metrology, Evaluation of Measurement Data-Guide to the Expression of Uncertainty in Measurement (JCGM 100:2008, Bur. Intl. Poids et Mesures, Sèvres, 2008).

${ }^{32}$ Y. Salissou and R. Panneton, "Quantifying the through-thickness asymmetry of sound absorbing porous materials”, J. Acoust. Soc. Am., Volume 124(2), EL28 (2008).

${ }^{33}$ O. Doutres, N. Atalla, and H. Osman, "Transfer matrix modeling and experimental validation of cellular porous material with resonant inclusions," J. Acoust. Soc. Am., Volume 137, no. 6, 3502-3513 (2015).

${ }^{34}$ O. C. Zwikker and C. W. Kosten, Sound-Absorbing Materials (Elsevier, Amsterdam, 1949). 